\title{
Occurrence of Guillain-Barré syndrome and Myasthenia Gravis in an elderly male
}

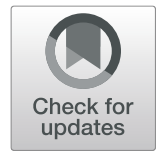

Madhavi Karri ${ }^{*}$ D, Balakrishnan Ramasamy and Santhosh Perumal

\begin{abstract}
Introduction: The occurrence of both Guillain-Barré syndrome(GBS) and myasthenia gravis (MG) in the same individual is rare. The underlying pathophysiology was assumed to be autoimmune humoral mechanisms and molecular mimicry with a cross-reaction between autoantibodies and myelin sheath of peripheral nerves and acetylcholine receptors of the neuromuscular junction (NMJ).

Case description: A 68-year-old male known diabetic and hypertensive with good drug compliance presented with acute onset quadriparesis with bulbar involvement for 1 day. On examination, he had mild neck flexion weakness and bulbar weakness. He had flaccid quadriparesis with absent deep tendon reflexes and negative Babinski. The rest of the neurological examination was normal.

Discussion and evaluation: Blood and electrophysiological studies showed evidence of demyelinating polyradiculoneuropathy with temporal dispersion suggestive of Guillain-Barré syndrome. He was treated with intravenous immunoglobin and complete resolution of symptoms. Two months later, he presented with new-onset asymmetrical ptosis and bulbar symptoms for 3 days. On further evaluation, repetitive nerve stimulation showed postsynaptic neuromuscular disorder pattern typical of myasthenia gravis, which was further confirmed by positive acetylcholinesterase receptor antibodies (AChR). Thymoma was ruled out by imaging. He was treated with anticholinesterases, low dose steroids, and immunosuppressants (azathioprine) following which he had improvement of symptoms.
\end{abstract}

Conclusion: Our patient, who was initially diagnosed with Guillain-Barré syndrome and recovered, presented 2 months later with a new-onset illness suggestive of myasthenia gravis. This existence of two different neurological entities in the same individual is a rarity, and early recognition is essential for treatment decision and prognostic strategies.

Keywords: Guillain-Barré syndrome, Myasthenia gravis, Immunosuppressants

\section{Introduction}

Guillain-Barré syndrome (GBS) and myasthenia gravis (MG) are two different disease entities of the peripheral nervous system. Guillain-Barré syndrome is also known as an acute demyelinating inflammatory polyneuropathy characterised by acute symmetrical ascending motor weakness with areflexia with or without sensory involvement occurring secondary to autoantibodies against peripheral myelin. Myasthenia gravis is an autoimmune disease characterised by antibodies directed against acetylcholine receptors of the postsynaptic membrane of the neuromuscular junction. Clinically, it affects skeletal muscles, more commonly being ocular, bulbar, and respiratory muscles. It is insidious in onset and causes diurnal fluctuating weakness. Both diseases have different pathophysiology and the nature of the presentation. The appearance of both in the same individual is a rare entity and is often under-recognised [1]. So far, only 14 reports have been reported in which 15 cases have been diagnosed with the coexistence of MG and GBS. Here, we present a case of occurrence of GBS and MG in the same individual.

\footnotetext{
* Correspondence: dr.madhavikarri@gmail.com

Department of Neurology, PSG Institute of Medical Sciences and Research, Peelamedu, Coimbatore, Tamil Nadu 641004, India
} 


\section{Case description}

A 68-year-old male presented with acute onset paraesthesias of both upper limbs and lower limbs followed by difficulty in getting up from a squatting position for 1 day. He also had complaints of difficulty in swallowing more for liquids since then. He presented to our hospital walking independently. There was no history of preceding fever, loose stools, or upper respiratory illness. He was a known diabetic for 10 years and hypertensive for 2 years with good drug compliance and history of recurrent stroke (twice once in 2007 and other in March 2017) in the past recovered with no residual deficits. At the time of presentation, he was conscious, well oriented, and communicating well with normal speech. Spino-motor examination showed a flaccid quadriparesis of power 3/5 of all four limbs along with neck and truncal muscle weakness. Upper limbs were more affected than lower limbs with proximal weakness more than distal. Deep tendon reflexes were absent with bilateral plantar flexor. Gag reflex was weak. His SBC (single breath count) was 15 . Other cranial nerves and sensory system examinations were normal. No evidence of meningeal signs. With a working diagnosis of Guillain-Barré syndrome, he had been evaluated for the same.

His complete blood picture (haemoglobin-14 g/dL; total leucocyte counts-7100 cu $\mathrm{mm}$; platelets-154, $000 / \mu \mathrm{L})$ was normal. Erythrocyte sedimentation rate was $27 \mathrm{~mm}$ after $2 \mathrm{~h}$. Chest X-ray and urine analysis were normal and showed no evidence of infection. His sugars were $97 \mathrm{mg} / \mathrm{dL}$. His renal functions and liver functions were normal. Serum electrolytes (sodium-135 mEq/L; potassium $-3.79 \mathrm{mEq} / \mathrm{L} ;$ ionised calcium-1.201 mmol/L; magnesium-1.8 mEq/L; phosphorus-2.6 mg/dL) were normal. His thyroid functions (serum TSH-3.5 mIU/L; free T4-1.8 ng/ $\mathrm{dL}$ ) were normal. Magnetic resonance imaging (MRI) of the brain with whole spine screening was normal. Serum CPK levels were not significantly elevated (414 $\mathrm{U} / \mathrm{L})$. We used Nihon Kohden with model number Neuropack X1 MEB - 2300K, Nihon Kohden Corporation, Japan, for nerve conduction studies (NCS). Nerve conduction studies (NCS) of all four limbs were suggestive of severe demyelinating neuropathic changes with temporal dispersion-considering all the features favouring the diagnosis of Guillain-Barré syndrome (GBS). He was treated with intravenous immunoglobulin (IVIg) given for 5 days according to weight base dose after obtaining informed consent. $\mathrm{He}$ had worsening bulbar symptoms on the second day of hospital stay for which he was intubated to secure airway and put on mechanical ventilatory support. Cerebrospinal fluid (CSF) analysis was acellular with mildly elevated proteins. After the complete course of IVIg, he was gradually weaned off from the ventilator by the seventh day and also had improvement of symptoms. He had autonomic symptoms of hypothermia, profuse sweating, and paralytic ileus during the ICU stay, which resolved gradually after treatment with IVIg. He had persistence of hoarseness of voice for which ENT surgeon opinion for vocal assessment done showed mild bilateral restriction of vocal cord mobility with an ulcer over left posterior commissure secondary to intubation. He was able to eat and swallow orally with no signs of any aspiration, and his motor symptoms improved. He was able to walk without support and hence was discharged after 2 weeks of hospital stay.

He has again come to our outpatient clinic after 2 months with complaints of sudden onset of asymmetrical ptosis (left more than right) with bulbar symptoms (hoarseness of voice and nasal regurgitation to liquids) and dysarthria for 3 days since July 7, 2018. He had a history of upper respiratory tract infection for 3 days, 1 week preceding the present symptoms. On examination, he had bilateral asymmetrical ptosis (left more than right) with poor gag reflex and single breath count was 19 with the rest of the neurological examination normal. MRI brain plain was done, and acute stroke was ruled out. Blood investigations were normal. Nerve conduction studies of all four limbs showed evidence of axonal neuropathy in both lower limbs and demyelinating neuropathy in upper limbs with temporal dispersion. Serum CPK levels were normal. With a diagnosis of myasthenia gravisMGFA type IIB clinically-RNS showed postsynaptic neuromuscular disorder pattern classical for myasthenia gravis. Serum AChR antibodies were strongly positive $(15.9 \mathrm{nmol} / \mathrm{L})$. Contrast-enhanced computed tomography (CECT) of the thorax showed no evidence of thymoma. He was started on anticholinesterases, steroids (low dose), and immunosuppressants (azathioprine) following which he had an improvement in symptoms.

Here, our patient had initially Guillain-Barré syndrome who is treated and recovered completely; 2 months later, he has worsening bulbar symptoms with asymmetrical ptosis (left more than right). Additional investigations like RNS (done by RMS Portable Aleron, PEMGM2\122026\ADBX, Recorders and Medicare Systems Private Limited, India) showing postsynaptic disorder and strongly positive AChR antibodies were more favouring the diagnosis of myasthenia gravis.

\section{Discussion and evaluation}

GBS is an acute neurological illness of the peripheral nervous system characterised by ascending symmetrical paralysis with areflexia. Laboratory evidence supporting GBS was electrophysiological evidence of demyelinating 
neuropathy with prolonged latencies and absent $\mathrm{H}$ and $\mathrm{F}$ reflexes and albumin-cytological dissociation in CSF [2]. Myasthenia gravis is an entirely different entity. It is an antibody-mediated $\mathrm{T}$ cell-dependent immune reaction on proteins of the postsynaptic membrane of the neuromuscular junction. Fatiguability and weakness of the skeletal muscles clinically characterise myasthenia gravis. It is usually of clinical suspicion and confirmed electro-physiologically by repetitive nerve stimulation with fatiguability pattern and identification of autoimmune antibodies in the blood against acetylcholine or muscle-specific kinase receptors [3]. The occurrence of both in the same individual is a rare entity. The mechanism for the existence of these two diseases is that they share common autoimmune humoral mechanisms and underlying molecular mimicry. There is a cross-reaction between the autoantibodies and myelin sheath of peripheral nerves and acetylcholine receptors of the neuromuscular junction [4]. Presentation of both in the same individual is unusual. The incidence of MG and GBS was reported to be about $0.4-1.7$ and $10-20$ per million persons per year accordingly. In Kizilay's and Kraus's reports, post-infectious GBS was followed by MG by 3 weeks and 10 weeks, respectively $[5,6]$.

In a case analysis of the clinical features of combined Guillain-Barré syndrome and myasthenia gravis from 1960 to 2012 done by Hsieh and his colleagues, 12 cases were identified with the concurrence of GBS and MG [7]. Out of which, four had post-infectious Guillain-Barré syndrome followed by the development of myasthenia gravis concurrently or concomitantly within 1 month with all cases having ptosis and areflexia. The other common presentations were limb weakness, oculo-bulbar weakness, and respiratory involvement. Functional outcome was mentioned in 10 patients, and seven had a good result (Hughes scale $\leq$ 2). Detection of ptosis with or without ophthalmoplegia, distribution of limb weakness, and reflexes can help in recognising combined Guillain-Barré syndrome and myasthenia gravis. The occurrence of ptosis, dysphagia, and paradoxical weakness of respiratory muscles (dyspnoea) after the resolution of symptoms with prior GBS suggests affection of neuromuscular junction [4]. The early recognition of this combination of the peripheral nervous system and neuro-muscular junction inflammation is essential for initial treatment and long-term prognosis. Management of MG and GBS acutely include plasmapheresis or IVIg. These are temporary modalities to treat the acute stages. Though both are equally effective, plasma exchange is considered more effective [8]. The patient had to be gradually initiated with high or low dose steroids with immunosuppressants for MG though it is not indicated in GBS. It was thought that the autoimmune process and presence of similar proteins in both disease processes can cause identical clinical picture and their response to acute therapy.

\section{Conclusion}

GBS and MG are two different neurological entities with different pathophysiology. New onset or persistence of symptoms in a patient with GBS treated with IVIg or plasmapheresis should probe for another autoimmune neurological illness, preferably autoimmune neuromuscular disorders like myasthenia gravis.

\section{Abbreviations}

CECT: Contrast-enhanced computed tomography; CPK: Creatine phosphokinase; CSF: Cerebrospinal fluid; GBS: Guillain-Barré syndrome; IVIg: Intravenous immunoglobulin; MG: Myasthenia gravis; MRI: Magnetic resonance imaging; NCS: Nerve conduction studies; RNS: Repetitive nerve stimulation; SBC: Single breath count

\section{Acknowledgements}

Not applicable

\section{Authors' contributions}

MK has contributed to the concept, design, analysing and interpreting of the data, and drafting and revision of the article. BR analysed and interpreted the data and drafted and revised the article. SP analysed and interpreted the data and drafted and revised the article. All authors read and approved the final manuscript.

\section{Funding}

Nil sources of funding

Availability of data and materials

Corresponding author, Department of Neurology.

\section{Ethics approval and consent to participate}

Obtained. Approved by PSG Institute of Medical Sciences and Research Institutional Human Ethics Committee Recognized by The Strategic initiative for Developing Capacity in Ethical Review (SIDCER) 08/10/2019. Project no: 19/279. Ref no: PSG/HEC/2019/Appr/Exp/253.

Consent for publication

Not applicable

Competing interests

The authors declare that they have no competing interests.

Received: 15 October 2019 Accepted: 2 December 2019

Published online: 12 December 2019

\section{References}

1. Zhang J, Niu S, Wang Y, Hu W. Myasthenia gravis and Guillain-Barré cooccurrence syndrome. Am J Emerg Med. 2013;31(8):1264-7.

2. van Doorn $P$, Ruts $L$, Jacobs B. Clinical features, pathogenesis, and treatment of Guillain-Barré syndrome. Lancet Neurol. 2008;7(10):939-50.

3. Romi F, Gilhus NE, Aarli JA. Myasthenia gravis: clinical, immunological, and therapeutic advances. Acta Neurol Scand. 2005;111(2):134-41.

4. Kung SL, Su JM, Tsai SJ, Lu TM, Chen CM. Concurrent Guillain-Barre syndrome and myasthenia gravis:the first case in Taiwan. Acta Neurol Taiwan. 2009;18(3):193-7.

5. Kizilay F, Ryan HF, Oh SJ. Myasthenia Gravis and Guillain-Barré syndrome occurring simultaneously in the same patient. Muscle Nerve. 2008;37(4):544-6.

6. Kraus J, Teismann I, Kellinghaus C, Duning T, Ringelstein EB, Nabavi DG, et al. Temporal coincidence between AMAN type of GBS and myasthenia gravis. J Neurol. 2007;254(2):264-5. 
7. Hsieh M-Y, Chan O-W, Lin J-J, Lin K-L, Hsia S-H, Wang H-S, et al. Combined Guillain-Barre syndrome and myasthenia gravis. Brain Dev. 2013;35(9):865-9.

8. Misra I, Temesgen FD, Soleiman N, Kalyanam J, Kurukumbi M. Myasthenia gravis presenting like guillain-barre syndrome. Case Rep Neurol. 2012;4(3): 137-43.

\section{Publisher's Note}

Springer Nature remains neutral with regard to jurisdictional claims in published maps and institutional affiliations.

Submit your manuscript to a SpringerOpen ${ }^{\circ}$ journal and benefit from:

- Convenient online submission

- Rigorous peer review

- Open access: articles freely available online

- High visibility within the field

- Retaining the copyright to your article

Submit your next manuscript at $\boldsymbol{\sim}$ springeropen.com 